\title{
Implementation of Energy Efficient Models using MATLAB for Wireless Sensors Network Protocols
}

\author{
Gudimella Krishna Kishore, Gollakota Venkata Krishna Sharma, Bonthu Brahma Reddy
}

\begin{abstract}
Wireless era is the most developing technology in this era and used in many actual time applications; as an instance, our everyday usable Smartphone's lie in wireless era. These days the variety of humans die from style of illnesses like heart attack, blood strain, allergies, cardiovascular dieses, etc. It is thrilling to apply Wi-Fi era, that's aggregate of wireless networking and Wi-Fi communication to boom the lifestyles of humankind through technique named Wi-Fi frame region networks (WBAN). The distinctive form of sensor nodes is connected in and across the human frame to feel the modifications, fetch the statistics and skip the fetched records to the server that's inside the far flung region for far off fitness monitoring purpose.

Loss while consuming the different Energies in network or via nodes is in the running situation, the actual data transmission will take region. Another factor that we initiate the required preferences which are obligation cycles. It is the time taken via the sensor nodes that perform irregularly in preference to continuously. When the transmission distance may be very small in comparison to the threshold distance, obligation cycle may be very high, and for this reason, to get better prices for battery for the fact of ideal time, and consequently, battery recovery effect is not taken into consideration. This paper focused on techniques designing a network location having duration and width of length $10 \times 10$ along with 20 numbers of sensor nodes for special transmission distance modifications from $10-1500$ for one of a kind bit quotes. All the simulation tactics could be done in $M A T L A B$ surroundings, and the performance parameters like CDF, postpone spread, path loss Duty cycle and Energy consumption with baseline. offline, and recovery set of rules may be measured along with optimization algorithm named as Hybrid-Genetic algorithm.
\end{abstract}

Keywords : CDF,MATLAB,WBAN. Wi-Fi.

\section{INTRODUCTION}

Evolution of wireless, scientific and laptop networking technology have specifically modeled to emerge in novel scenario of science and technology referring to as On Body Wi-Fi devices (WBANs).In accordance, with application for On-Body W-devices aren't confined to the medical discipline. WBASN is likewise considered as a vital section

and part and parcel of Sensing Networks Wirelessly, due to its on demand devices requirements in real time scenarios. In

Revised Manuscript Received on December 13, 2019.

* Correspondence Author

Gudimella Krishna Kishore *, Research Scholar, Dept of ECE, Gitam Deemed to be University, Vishakhapatnam, India. Email: krishnakishore414@gmail.com

Assistant Professor, Dept of ECE, Vignana Bharathi Institute of Technology, Hyderabad, India.

Gollakota Venkata Krishna SHARMA, Professor, Dept of ECE , Gitam Deemed to be University, Vishakhapatnam, India. Email: sharma.gollakota@gitam.edu

Bonthu Brahma Reddy, Professor, Dept of ECE, Vignana Bharathi Institute of Technology, Hyderabad, India. Email: reddybb@hotmail.com each On Body Wi-Fi devices and Sensing Networks Wirelessly, factors related to elimination of residual energies, mobility and nodes to any senor network confinement is a phenomenal concept to improvise higher optimization of WSNs. Our design model [1] proposes a generic scenario to obtain set of rules to obtain the localization of sensor nodes.

In On Body Wi-Fi devices, diminishing and congruence for network are top notch parameters. WBANs encompass 3 stages; First degree was to enable lesser strength in sensors-nodes where each such elements can be energized with battery and performs specific operations in certain period of space-time interval unable of re-charging and batteries and their substitutes. Currently, predicted-nodes can be positioned on the individual-body, across the frame or incorporated with the organs of individuals-body. The 2nd stage would define a principle node where each gateway or coordinator are being controlled by toddler (substitutive) nodes, where the requirements to provide and sustain energy would be minimized for nodes considering its design and versatility. The third level is the neighborhood or net network that enables for tracking scenarios. Intake of different energies with respect to one or more deices are the prime requirements for On body chip Wi-Fi based devices as the limited resource for batteries.

In [2], [3] most of the troubles/problems are associated to enhance and impart the growth of the designed network, However the prime desirable-layer for discussing electricity and energy problems is in media access control layer. Prime aspect of saving strength or improving power expertise, reduce the energy wastage. There are numerous assets of power wastage which include packet collision, overhearing, idle listening, control packet overhead, and so on. Major source of electricity inefficiency most of the required and user-mentioned assets is tend to provide packet collision for on body chip devices controlled wirelessly. Collision indulging in power related expertise parameters (input and output latency), high-productivity, and reliability in accessing communications throughout the network, are simple essential needs in the layout of media access control protocol. The essential manner of saving energy or enhancing electricity performance is to minimize energy residual loss in the network and the nodes. Simulations on MATLAB have been implemented for exceptional eventualities to evaluate and analyze course loss. Simulations-Results display that route loss is maximum in frame-in sequence data-comm, as compared to frame-on and frame-off conversation and additionally plot consequences with the energy consumption via .

Published By: 


\section{Implementation of Energy Efficient Models using MATLAB for Wireless Sensors Network Protocols}

\section{RELATED WORK}

Chen fu Yi [16] has described Power-Energy emphasized for prime criteria's relating theoretical energies governing for wireless sensor nodes, particularly for $\mathrm{Wi}-\mathrm{Fi}$ frame place networks (WBAN) where the sensory function provide a particular acceptable location in-out in the human-internals. In the article, system architect provides a system which would intake energy to initiate a model related to data-transmission distanced and transmission statistics fee over on-frame Wi-Fi verbal exchange hyperlink.

S. Ahmed [17] has defined the role of the Wi-Fi frame network (WBN) and its growth in fitness carrier centers. WBN also helps to enhance human health. By conducting a comprehensive survey, it's far envisioned that Virtual Doctor Server has existed in WBAN architecture.

LamiaChaariFourati [18] proposed on body chip devices being implemented in clinical health based care designs. Frequently, numerous programs which can be advanced in BAN in WSN (Wireless Sensor Network) technology. Ramli, S.N.

[19]has described wireless conversation technologies and sensors which might be developing regularly and empowering the improvement of On Body Wi-Fi devices (WBAN). The motive at the back of using wireless technology are new, realistic and creative thoughts offers by using specific health-based sensors which improvises to affect the fitness visualization and tracking, in different fitness care scenarios.

C Li [20] has defined a Wi-Fi body vicinity community with brilliant impact on our day by day existence. As time passes, it has end up a crucial a part of our life. WBAN also offers the answer to continual infection as well as the patients which might be tormented by constant and long term ache.

Emmanuel Davies [21] has centered at the most rising era of nowadays on chip bogy devices would pasteurize with its packages in diverse regions in certain complex areas. OBCDs are relevant in diverse healthcare services and presenting medicinal drug to lessen the requirement of caregivers. OBCD's provides the people \{elderly and sic\} to increase the span of the chances to live diversely.

Sapna Singla et al [22] has explained the concept of wireless frame vicinity community as the mostly drifted in vicinity of current problem associated. Noticeably, this design been used in numerous packages. Preventive and improvising therapy scenarios which have modeled to affect the changes while using OBCD.

Pervez Khan et al [23] improvised on sensor network on media access protocol with IEEE standards $\{15.6\}$ in a quick region of noticeably low power-transit. Novel modeling for accessing devices wirelessly with body on location network scenarios covering higher records and costs which could get entry to the information in location of, or interior a human body.

Xin Qi et al [24] has discussed wireless body vicinity community as an emerging subject nowadays which gives approach to the cell fitness i.e. M-health. Basically, on body chip Wi-Fi devices is used to accumulate information from in-body or around frame sensors nodes.

\section{MATHEMATICAL ANALYSIS FOR ENEGY CONSUMPTION MODEL WSN}

To provide an estimated energy consumption with a time frame interval $\mathrm{T}$ we initiate certain number of random events occurring in time frame $\mathrm{T}$ with distribution considered as Poisson and governing factor $\alpha$ as:

$P(k, t)=\frac{(\alpha . t)^{k} e^{-\alpha t}}{k !}$

Hence, consumed estimated energy is given by

$$
\begin{gathered}
E_{\text {node }_{i}}=\beta\left[\sum_{k=0}^{\alpha}\left(\frac{(\alpha \cdot t)^{k} e^{-\alpha t}}{k !}\right) \cdot(\gamma-k) \cdot E_{R x}+k \cdot E_{T x}\right. \\
\left.+k \cdot\left(E_{R T T}+E_{T T R}\right)\right]+\gamma \cdot E_{f}
\end{gathered}
$$

Where

$$
\begin{gathered}
\beta \in N^{*}-\{1\} \text { if } \text { node }_{i} \text { is an intersection point } \\
\beta=1 \text { else } \\
E_{T x}=\text { Enenrgy of Transsmission } \\
E_{R x}=\text { Enenrgy of Recption } \\
E_{T T R}=\text { Enenrgy of Transition form } T x \text { to } R x \\
E_{R T T}=\text { Enenrgy of Transition form } R x \text { to } T x \\
E_{f}=\text { Enenrgy ofoperation } \\
k=\text { number of events occured } k=0,1,2,3, \ldots N \\
\gamma=\text { sup }\left(T / T_{u}\right)
\end{gathered}
$$

To provide a realistic model for energy consumption we need to consider the consumption of the energy where event occurrence for the time interval would depend on energy for the operation of the realistic sensors nodes also the reception energy for each scenario considered. Having thought of the consumption energy we have taken criteria of transmitter related energies for each scenario of the sensing the data and its real time implementations.

\section{SIMULATION MODEL}

In this research, we have provided wireless body location community the usage of genetic set of rules to decrease the power intake. The maximum critical benefit of the H-GA is their capacity to apply accumulative statistics approximately the preliminary unknown seek area so as to flow the subsequent searches in to beneficial areas [8]. A On body chip WIF devices (WBAN) connects impartial nodes (Gravity Sensors and sensor actuators) have been situated within the garments, at the body or below the skin of a person. On body chip WIFI devices inculcates different scenarios on current trend of applications in domestic/fitness care, medication, sports, multimedia, and lots of other regions [9]. To design and increase the proposed paintings, underneath steps has been followed:

1: Initialization of the Wireless frame vicinity community with peak and width $(10 * 10)$ is the number one step.

2: In the simulated network considered implementing the sensor nodes are positioned in the region of interest.

3. Based on the position paths are identified and accordingly source and destinations nodes for each path and positions are estimated. 
4: The optimization algorithm implemented based of improved (Hybrid model) genetic scenario for the paths and nodes inside/outside the network.

5: Optimization of path inside the network is taken area the usage of genetic set of rules

6: Calculate the parameters namely, CDF, delay spread, path loss, energy intake, transmission time and responsibility cycle.

WBAN systems can capitalize on current technological improvements have been 284 modeled and opted for novel methods for human-behavior and motion, making prolonged interest analysis more feasible [10].The overall performance parameters which have been measured are listed under:

\section{A. Duty Cycle:}

A biosensors implanted on the human frame will function as specific cycle which would estimate the on and off times known to be duty cycle and is the primary parameter for energy consumption contrast of the distinct MAC protocols, due to the fact it's long reaches are dependent minimal base on the trans receiver used [11].The discern is shown under: Here, the active mode might be determined earlier than the gathered at transmission and base station is denoted as $\mathrm{T}$ _ac this is called lively mode and Tsc is referred to as sleep mode. Since, the principle aim of MAC protocol layout is to lessen energy consumption, at the identical time as supporting brilliant scalability and collision avoidance. Currently, working on the designed protocol where the lessen strength would intake from all the resources that we've were given recognized determine the loss in energy via nodes and network i.e. idle listening, node or data collisions, packet-overhead and manipulate overhead [12]. In the active mode of the biosensors, the uncooked records is surpassed to the filtrations and amplification process. After that, the sign has modulated the usage of a few modulation strategies after which transmitted to e-CPU through the wireless community. After the transmission of the data, the biosensors may visit its SLEEP mode having period Tsl. In the responsibility cycles especially 3 steps are taken into consideration named as Active mode, Sleep mode and Transient mode.

$$
T_{\text {Transient }} \text { Mode }=T_{s l \rightarrow a c}+T_{a c \rightarrow s l}
$$

The everyday cost of the short mode is smaller than the Active mode. This is the time occupied by way of the cycle of operation of a simulator. The sensor node affects the system's strength intake through way of converting its obligation cycle [13]. Particularly, its miles defined within the shape of percent time or in the shape of ratio. It is the proportion of time thru which a transmission is accomplished.

\section{B. Energy Consumption:}

Energy consumption is the defined as the total amount of energy being ate up by using every node in on chip Wi-Fi devices at special-layers in the considered network of the design opted. The energy consumed till now is estimated via summation in each operation mode throughout simulation time. It is described mathematically as underneath:

$$
\text { Energy Consuption }=\sum_{i=0}^{n-1} \text { Energy_each_node }(i)
$$

It is the count number of total electricity that is elapsed in the course of the transmission of packet statistics from one sensor to any other sensor node. The capacity for which the transmitter would provide the packet transfer from source-node to final-destination nodes of the Network considered. The electricity intake model characterizes the sources of electricity consumption in the community [14].In simulation work, strength exists in distinctive bureaucracy like transmission electricity, receiving power and energy of operation. In accordingly we have mention in the proposed scenario where the power consumed by the network is directly proportional to the integration of all the energies at or in the network.

\section{Transmission Time:}

Probability of estimation of minimum time required to send single or multiple packets in a definite time interval required as known to be transmission time represented as megabits. The bit structure is determined by parameters:

\section{SIMULATION RESULTS}

As per the design criteria we have observed different equations for Energy calculations as per the no of nodes and estimating the minimum probability for the least energy per node.

We propose a simulation environment in current design model where each nodes are clustered with its energy efficient model and its distances from the reference. To enable such scenarios we have considered 10 nodes from the network size of $[10,10]$ and $[20,20]$ with minimum energy of 0.5 watts.

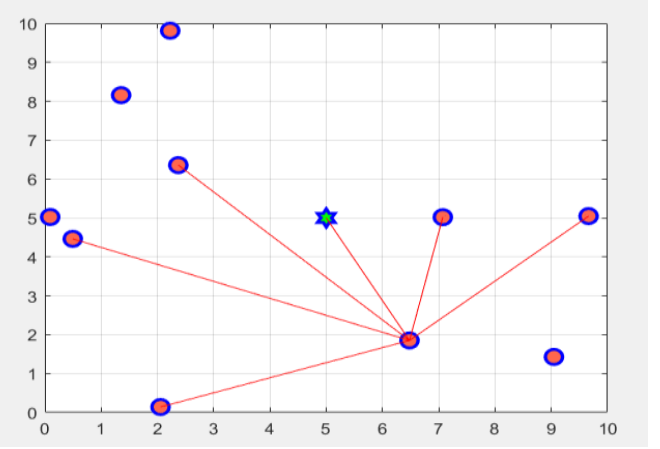

Fig.1. Describes about sensor nodes and its connected nodes.

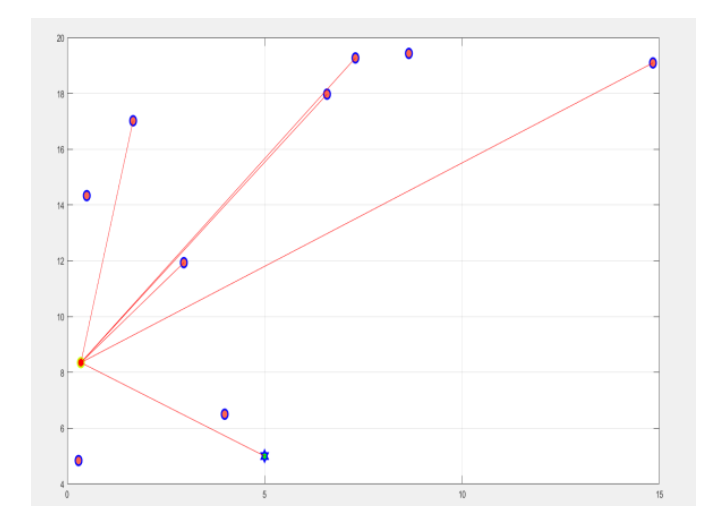

Fig.2. Describes about sensor nodes and its connected nodes and cluster head. Blue Eyes Intelligence Engineering \& Sciences Publication 
Implementation of Energy Efficient Models using MATLAB for Wireless Sensors Network Protocols

\section{Energy Efficient Plots:}

For each relative changes on distances and node energies the plotting for $\mathrm{x}$-direction and $\mathrm{y}$-direction is implemented accordingly.

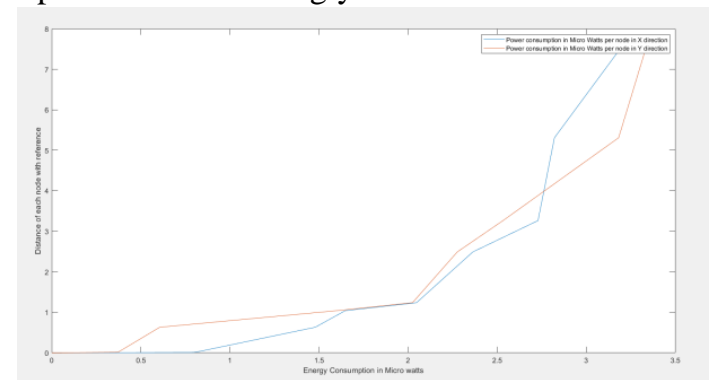

Fig.3. Energy values for sensor nodes and its connected nodes in $x$ and $y$ directions for $[10,10]$.

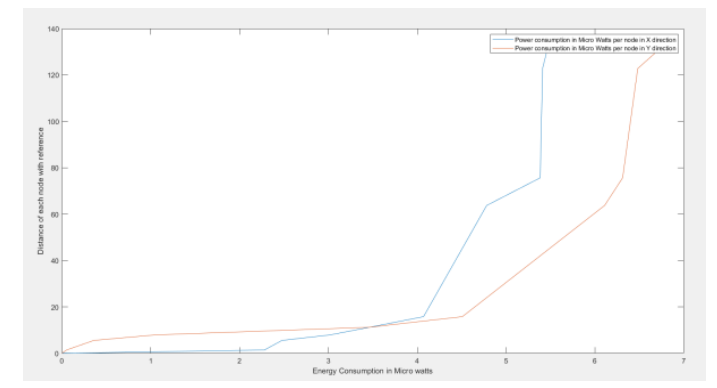

Fig.4. Energy values for sensor nodes and its connected nodes in $x$ and $y$ directions for [20, 20]

Table-I: Representing the Performance calculated parameters for Proposed Model.

\begin{tabular}{|l|l|l|}
\hline SNo & \multicolumn{1}{|c|}{$\begin{array}{c}\text { Performance } \\
\text { Parameters }\end{array}$} & \multicolumn{1}{|c|}{ Status } \\
\hline 1. & $\begin{array}{l}\text { Energy consumption of } \\
\text { each Tx and Rx phase }\end{array}$ & $\mathbf{0 . 5} \mathbf{~ W W}$ \\
\hline 2. & $\begin{array}{l}\text { Energy consumption of } \\
\text { each packet }\end{array}$ & $\mathbf{0 . 3 2} \boldsymbol{\mu \mathbf { w }}$ \\
\hline 3. & Rounds & $\mathbf{3 0 0 0}$ \\
\hline 5. & No of Nodes Alive & $\mathbf{9}$ \\
\hline 6. & $\begin{array}{l}\text { Total Nodes per }[10,10] \\
\text { and [20,20] }\end{array}$ & $\mathbf{1 0}$ \\
\hline 7. & Dead Nodes & $\mathbf{1}$ \\
\hline
\end{tabular}

Our design is estimated with [10,10] and [20,20] model for each 10 sensor nodes where only one dead sensor node was found and Estimated energy for each Node was given with $0.5 \mathrm{~W}$ supply. For Each scenario we have estimated our design model dead and alive node from figure 1 and 2.

\section{Conclusion}

In this research work, we have proposed a scheme along with an energy consumption model with different transmission distance and transmission data rate in bps. The whole system has been designed for a wireless body area network that finds application in health care. WSN is a network in which sensors are powered by limited energy batteries and thus, energy consumption becomes an important factor. In the proposed work, we have considered three different schemes named as baseline, offline and recovery schemes and then find the energy consumption for every scheme. Here, for optimization Hybrid Genetic algorithm has been used. The simulation is carried out in MATLAB simulator having a WBAN of size length and breadth $=100 \times 100$ for 10 number of nodes.

\section{ACKNOWLEDGMENT}

The following work has been carried out using computational facility provided by DST's FIST lab in VBIT, Hyderabad. India.

\section{REFERENCES:}

1. Yick, Jennifer, Biswanath Mukherjee, and DipakGhosal, "Wireless sensor network survey", Computer networks, Vol.52, No. 12, pp. 2292-2330,2008.

2. Perrig, Adrian, John Stankovic, and David Wagner, "Security in wireless sensor networks", Communications of the ACM 47, no. 6 (2004): 53-57, 2004.

3. Bulusu, Nirupama, and Sanjay Jha, "Wireless Sensor Network Systems. A Systems Perspective (Artech House Mems and Sensors Library)", Artech House, Norwood, MA, 2005.

4. Otto, Chris, et al. "System architecture of a wireless body area sensor network for ubiquitous health monitoring." Journal of mobile multimedia 1.4 (2006): 307-326.

5. Latré, Benoît, et al. "A survey on wireless body area networks." Wireless Networks 17.1 (2011): 1-18.

6. Akkaya, Kemal, Mohamed Younis, and MeenakshiBangad. "Sink repositioning for enhanced performance in wireless sensor networks." Computer Networks 49.4 (2005): 512-534.

7. Jourdan, Damien B., and Olivier L. de Weck. "Layout optimization for a wireless sensor network using a multi-objective genetic algorithm." Vehicular technology conference, 2004. VTC 2004-Spring. 2004 IEEE 59th. Vol. 5. IEEE, 2004.

8. Azadeh, Ali, et al. "Integration of artificial neural networks and genetic algorithm to predict electrical energy consumption." Applied Mathematics and Computation 186.2 (2007): 1731-1741.

9. Reusens, Elisabeth, et al. "Characterization of on-body communication channel and energy efficient topology design for wireless body area networks." IEEE Transactions on Information Technology in Biomedicine 13.6 (2009): 933-945.

10. Jovanov, Emil, et al. "A wireless body area network of intelligent motion sensors for computer assisted physical rehabilitation." Journal of NeuroEngineering and rehabilitation 2.1 (2005): 6.

11. Marinkovic, StevanJovica, et al. "Energy-efficient low duty cycle MAC protocol for wireless body area networks." IEEE Transactions on Information Technology in Biomedicine 13.6 (2009): 915-925.

12. Ye, Wei, John Heidemann, and Deborah Estrin. "An energy-efficient MAC protocol for wireless sensor networks." INFOCOM 2002. Twenty-First Annual Joint Conference of the IEEE Computer and Communications Societies. Proceedings. IEEE. Vol. 3. IEEE, 2002

13. Jiang, Xiaofan, Joseph Polastre, and David Culler. "Perpetual environmentally powered sensor networks." Proceedings of the 4th international symposium on Information processing in sensor networks. IEEE Press, 2005

14. Chen, Yunxia, and Qing Zhao. "On the lifetime of wireless sensor networks." IEEE Communications letters 9.11 (2005): 976-978.

15. Montestruque, Luis Antonio, and PanosAntsaklis. "Stability of model-based networked control systems with time-varying transmission times." IEEE Transactions on Automatic Control 49.9 (2004): 1562-1572.

16. Chenfu Yi et. Al, "Energy Efficient Transmission Approach for WBAN Based on Threshold Distance", IEEE sensors journal, Vol. 15, No. 9, pp 5133-5141, Sep 2015

17. S.Ahmed et al.,"LAEEBA: Link Aware and Energy Efficient Scheme for Body Area Networks", IEEE, pp.435-440, May 2014.

18. LamiaChaariFourati "Wireless Body Area Network and Healthcare Monitoring System", Healthcare Informatics (ICHI), Page(s):362, Sept. 2014

19. Ramli, S.N, "Surveying the Wireless Body Area Network in the realm of wireless communication", IEEE, pp.58 - 61, 2011

20. C. Li et al, "Performance Evaluation of IEEE 802.15.4 for Wireless Body Area Network (WBAN)", IEEE, pp.1 - 5, 2009.

21. Emmanuel Davies, "A Survey on Wireless Body Area Network", International Journal of Scientific and Research Publications, Vol 4 Issue 3,Pp 1-7, 2014 
22. SapnaSingla et al, "A Review Paper on Wireless Body Area Network for Health Care Applications”, IJCSMC, Vol. 5, Issue. 10, pp.1 - 11, October 2016,

23. Pervez Khan et al, "Performance Analysis of WBAN MAC Protocol under Different Access Periods", International Journal of Distributed Sensor Networks, Vol. 11, no. 10,2015 .

24. Xin Qi et al, "MAC Protocol in Wireless Body Area Network for Mobile Health: A Survey and an Architecture Design", International Journal of Distributed Sensor Networks, Vol. 11, No. 10, 2015.

\section{AUTHORS PROFILE}

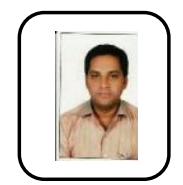

Gudimella Krishna Kishore has completed B.Tech and M. Tech in the year 2009 and 2011. Now Pursuing Ph.D. in Wireless Sensor Networks and VLSI Design from Gitam Deemed to be University under the superviosn of Dr. G.V.K. Sharma, Professor Department of ECE.

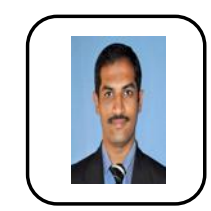

G.V.K. SHARMA obtained his Ph.D. and Master's degree from Andhra University and Indian Institute of Science, Bangalore respectively. Presently, he is a Professor in the Dept. of ECE, GITAM Deemed to be University, Visakhapatnam, India. His research interests include radar signal processing, VLSI signal processing and multicarrier communications.

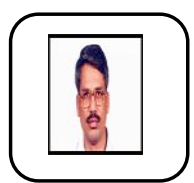

Bonthu Brahma Reddy has completed his M. Tech from IIT-Madras in the year 1982 and Ph.D. in the year 2016 from JNTUH. His research domain includes Wireless Sensors/Ad-Hoc Networks and Wireless Communication. 